ARTICLE

DOI: $10.1038 / s 41467-017-00209-z$

\title{
Aurora-B kinase pathway controls the lateral to end-on conversion of kinetochore-microtubule attachments in human cells
}

\author{
Roshan L. Shrestha1,4, Duccio Conti,2, Naoka Tamura ${ }^{1,5}$, Dominique Braun ${ }^{1}$, Revathy A. Ramalingam², \\ Konstanty Cieslinski ${ }^{3}$, Jonas Ries $\mathbb{B}^{3}$ \& Viji M. Draviam ${ }^{1,2}$
}

Human chromosomes are captured along microtubule walls (lateral attachment) and then tethered to microtubule-ends (end-on attachment) through a multi-step end-on conversion process. Upstream regulators that orchestrate this remarkable change in the plane of kinetochore-microtubule attachment in human cells are not known. By tracking kinetochore movements and using kinetochore markers specific to attachment status, we reveal a spatially defined role for Aurora-B kinase in retarding the end-on conversion process. To understand how Aurora-B activity is counteracted, we compare the roles of two outerkinetochore bound phosphatases and find that BubR1-associated PP2A, unlike KNL1associated PP1, plays a significant role in end-on conversion. Finally, we uncover a novel role for Aurora-B regulated Astrin-SKAP complex in ensuring the correct plane of kinetochore-microtubule attachment. Thus, we identify Aurora-B as a key upstream regulator of end-on conversion in human cells and establish a late role for Astrin-SKAP complex in the end-on conversion process.

\footnotetext{
${ }^{1}$ Department of Genetics, University of Cambridge, Cambridge CB2 3EH, UK. ${ }^{2}$ School of Biological and Chemical Sciences, Queen Mary University of London, London E1 4NS, UK. ${ }^{3}$ European Molecular Biology Laboratory, Cell Biology and Biophysics Unit, Meyerhofstrasse 1, Heidelberg, Germany. ${ }^{4}$ Present address: Center for Cancer Research, National Cancer Institute, National Institutes of Health, Bethesda, Maryland 20892, USA. ${ }^{5}$ Present address: Barts Cancer Institute, Queen Mary University of London, London EC1M 6BQ, UK. Roshan L. Shrestha and Duccio Conti contributed equally to this work. Correspondence and requests for materials should be addressed to V.M.D. (email: v.draviam@qmul.ac.uk)
} 
D uring cell division, accurate segregation of DNA requires the proper attachment of chromosomes to microtubules. Chromosome-microtubule attachment relies on a macromolecular structure-the kinetochore-that assembles on the centromeric region of chromosomes. We and others showed that kinetochores are predominantly captured along the walls of microtubules (termed lateral kinetochores) and then tethered onto the ends of microtubules (termed end-on kinetochores) ${ }^{1-4}$. This dramatic change in the geometry of kinetochoremicrotubule (KT-MT) interaction is achieved through a multistep end-on conversion process. End-on conversion is an indispensable process for lateral kinetochores: only when the ends of microtubules are tethered to the kinetochore, the growth and shrinkage of microtubule-ends (K-fibres) can impart pushing or pulling forces on the chromosome $\mathrm{e}^{5-7}$. Lesions in the end-on conversion process lead to defective chromosome segregation, as seen in cells lacking the loop region of the kinetochore protein $\mathrm{HEC} 1 / \mathrm{Ndc} 80^{4}, 8-13$, highlighting the importance of understanding how a lateral kinetochore is converted into an end-on kinetochore.

Several evolutionarily conserved kinetochore proteins are known to be important for forming mature attachments capable of load-bearing and end-on pulling events ${ }^{2-4}, 8,14-16$. Using deconvolution microscopy, we recently reported two markers to distinguish the plane of KT-MT attachment in human cells: (i) Mature end-on kinetochores, but not lateral kinetochores, recruit the Astrin-SKAP complex (ii) Mature end-on kinetochores, but not lateral kinetochores, are capable of converting the changes in K-fibre length into kinetochore movements ${ }^{4}$. However, upstream signaling pathways that control the endon conversion process have not been established so far in human cells. In yeasts, Aurora-B (Ipl1) kinase was shown to be an important upstream regulator of the end-on conversion process ${ }^{17}$. Whether Aurora-B plays a similar role in regulating the end-on conversion process in human cells is not known.

Distinct from the end-on conversion process that ensures the correct plane of KT-MT attachment, the error correction process ensures the correct orientation of attachment (referred as biorientation; reviewed in ref. ${ }^{9}$ ). Biorientation defects are resolved by Aurora-B kinase enriched at centromeres through feedback loops ${ }^{18-20}$; it phosphorylates outer-kinetochore substrates causing the detachment of non-bioriented KT-MT attachments (e.g., syntelic end-on attachments) ${ }^{16,} 21-27$. In addition, active Aurora- $B$ has been reported in human kinetochores during early mitosis ${ }^{28}$ and specifically on kinetochores that are laterally attached ${ }^{29}$. Whether Aurora-B at the outerkinetochore would destabilise immature lateral attachments is however not known.

Aurora-B and its counteracting phosphatases, PP1 and PP2A, are important for regulating outer-kinetochore assembly, KT-MT attachment stability, chromosome alignment and checkpoint function $^{29-38}$. Several Aurora-B counteracting phosphatases are recruited to the centromere and kinetochore in a temporally and spatially restricted manner (reviewed in refs 39,40 ). Whether Aurora-B counteracting phosphatases play a role in controlling the plane of KT-MT attachment remains unclear.

Here, we examine the role of Aurora-B kinase and its counteracting phosphatases in the end-on conversion process. We report that Aurora-B kinase impacts the end-on conversion process differently dependent on its sub-cellular localizationouter kinetochore vs. centromere. While Aurora-B targeted to the outer-kinetochore detaches lateral kinetochores prior to end-on conversion, Aurora-B targeted to the centromere stabilizes lateral kinetochores and retards end-on conversion. We find that lateral KT-MT attachments are relatively 'immune' to Aurora-B. Next, of the two Aurora-B-counteracting phosphatases, we find that
BubR1-associated PP2A, but not KNL1-associated PP1, is the most potent regulator of the end-on conversion process. Finally, we identify the Astrin-SKAP complex as a late player in the end-on conversion process. Thus, we report a novel spatially controlled role for Aurora-B in the end-on conversion process, establish BubR1-associated PP2A as a key phosphatase that counteracts Aurora-B activity during end-on conversion and finally, demonstrate a late role for Aurora-B regulated AstrinSKAP complex in the end-on conversion process. This study provides the first insight into how Aurora-B mediated signaling controls the plane of kinetochore-microtubule attachments in human cells.

\section{Results}

Aurora-B activity is high on immature lateral kinetochores. We first quantified and confirmed the presence of active Aurora-B on lateral kinetochores. For this purpose, HeLa cells were exposed to Monastrol to generate monopolar spindles, which mimic an early mitotic spindle configuration and allow clear distinction between lateral kinetochores and end-on kinetochores ${ }^{4}$. Immunostaining with antibodies against activating phosphorylation of Aurora-B (pThr232) (Supplementary Fig. 1A) showed that activated Aurora-B is abundant on lateral kinetochores $\left(n_{\mathrm{KTs}}>30 ; n_{\text {cells }}=10\right.$; Fig. 1a). Consistent with this finding, immunostaining with phospho-specific antibodies against Aurora-B substrate sites of the outer KT protein HEC1, Ser44 and Ser55 ${ }^{28}$, showed higher HEC1 phosphorylation on lateral compared to end-on kinetochores $\left(n_{\mathrm{KTs}}>20 ; n_{\text {cells }}=\right.$ 10; Fig. 1a,b). Thus, Aurora-B activity is high on lateral kinetochores.

Aurora-B at outer-kinetochore facilitate lateral kinetochores. To determine the contribution of outer-kinetochore associated Aurora-B activity in early mitosis, we locally increased Aurora-B activity at the outer-kinetochore by expressing the Mis12INCENP-GFP fusion protein, wherein a fragment of INCENP that lacks the centromere targeting domain but retains the Aurora-B docking domain is fused to the outer-kinetochore protein Mis $12^{26}$. As expected, but not reported so far, Mis12INCENP-GFP expressing HeLa cells showed high levels of HEC1pSer44 phosphorylation at all kinetochores (Supplementary Fig. 1B), confirming the fusion protein's ability to retain Aurora$\mathrm{B}$ activity at the outer kinetochore. Hence, this approach allowed us to quantify the effect of a localised increase in Aurora-B activity.

To study KT-MT attachment status (end-on vs. lateral) during early mitosis when end-on conversion events occur, we immunostained Monastrol-treated Mis12-INCENP-GFP expressing cells with antibodies against GFP, Tubulin and SKAP, a microtubule-end binding protein ${ }^{41,42}$ specifically enriched on end-on but not lateral kinetochores ${ }^{4}$ (Fig. 1c). In monopolar spindles of control cells, less than $25 \%$ of kinetochores were laterally attached and they lacked SKAP ${ }^{4}$. However, in Mis12-INCENP-GFP expressing cells, more than $95 \%$ of kinetochores were laterally attached (Fig. 1d,e) and they lacked SKAP $\left(n_{\text {cells }}=26 / 31\right)$ (Fig. 1d and Supplementary Fig. 1C). The striking defect in the plane of KT-MT attachment observed in Mis12-INCENP-GFP expressing cells was completely reversed following Aurora-B inhibition using ZM447439, as almost all kinetochores were attached to MT-ends and enriched for SKAP $\left(n_{\text {cells }}=20 / 20\right)$ (Fig. 1d,e and Supplementary Fig. 1C). Thus, retaining Aurora-B activity at the outer-kinetochore promotes the incidence of lateral kinetochores, suggesting a failure in the end-on conversion process. The inhibitor studies confirm that this failure is not due to non-specific interference by Mis12- 
a

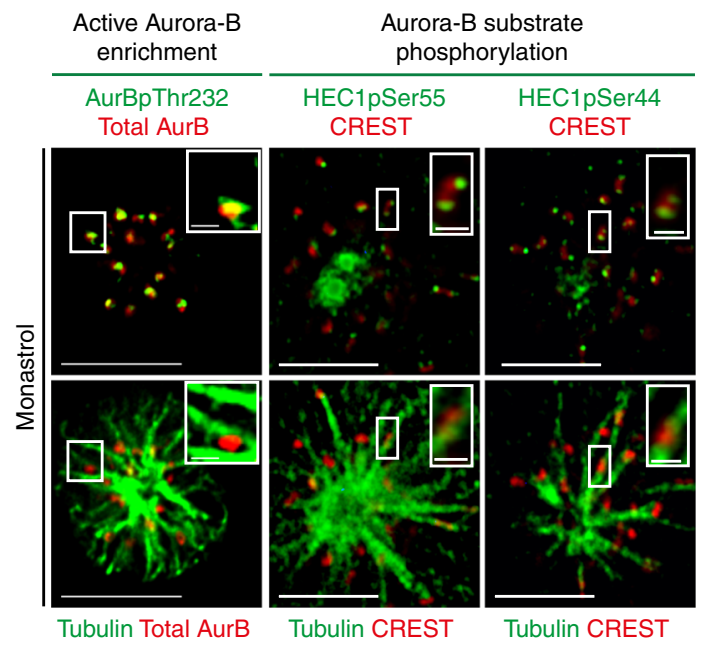

C

Experimental regime

Plasmid transfection

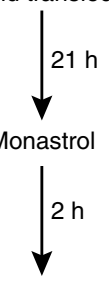

ZM447439/ DMSO

+Monastrol+MG132

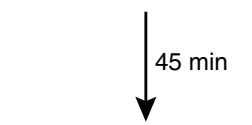

Immunostaining for KT-MT attachment status d

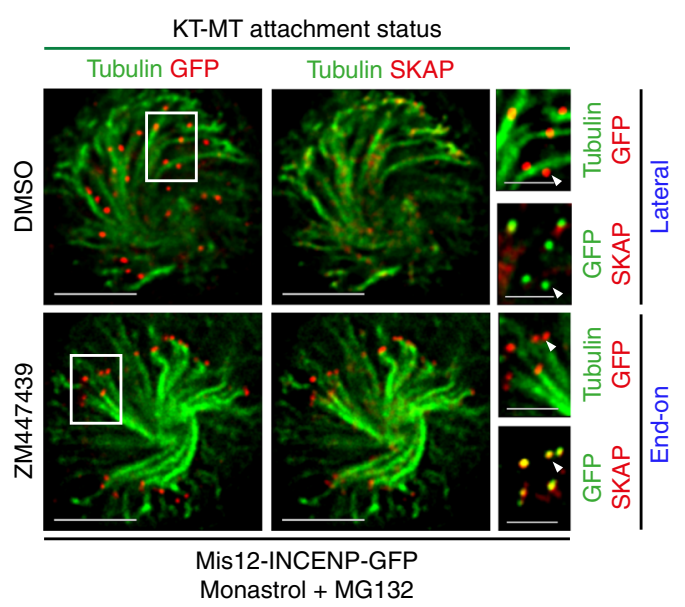

b

Extent of HEC1 phosphorylation

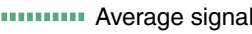

- HEC1pSer55/44 CREST

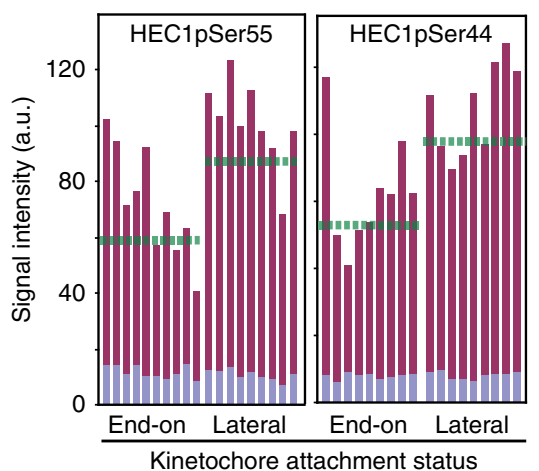

e

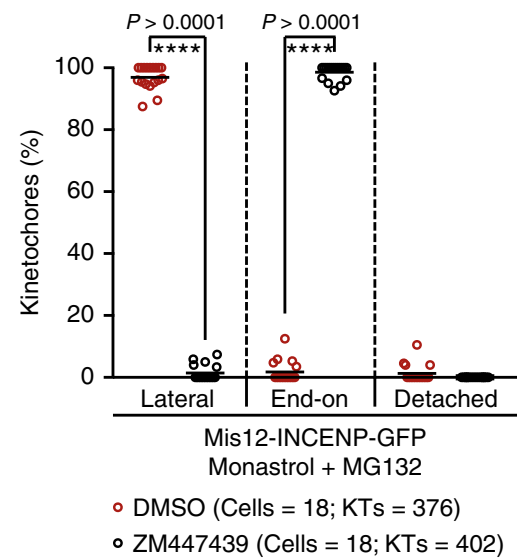

Fig. 1 High Aurora-B activity promotes KT attachment to MT-walls. a Representative images show high Aurora-B activity on lateral kinetochores. Monastrol treated cells were immunostained with antibodies against Tubulin, Aurora-BpThr232 and total Aurora-B (AurB) (left panel) or CREST antisera and antibodies against Tubulin and either HEC1pSer55 (middle panel) or HEC1pSer44 (right panel). Cropped images show lateral-kinetochores. Scale: 5 um in uncropped images; $1 \mu \mathrm{m}$ in cropped images. b Graphs show higher average signal intensities of HEC1pSer55 (left) and HEC1pSer44 (right) in lateral compared to end-on kinetochores as assessed from at least nine randomly chosen kinetochores from cells in a. CREST signal intensities are used as internal controls. c Experimental regime: Cells transfected with plasmid vectors encoding Mis12-INCENP-GFP were exposed to Monastrol and MG132 with either ZM447439 or DMSO (solvent control), prior to immunostaining. d Images of cells expressing Mis12-INCENP-GFP treated as in c and immunostained with antibodies against Tubulin, SKAP and GFP. White arrowheads in cropped images show 'Lateral' kinetochore lacking SKAP (upper panel) and 'End-on' kinetochore enriched with SKAP (lower panel). Scale: $5 \mu \mathrm{m}$ in uncropped and $2 \mu \mathrm{m}$ in cropped images. Boxed areas in a and $\mathbf{d}$ correspond to cropped images. e Graph shows percentage of lateral, end-on and detached kinetochores in Mis12-INCENP-GFP expressing cells treated as in c. Each circle represents value from one cell. Black horizontal bar marks average values from three independent experimental repeats. ${ }^{\star * \prime}$ indicates statistically significant difference on the basis of $P$-values obtained using unpaired Student's $t$-test

INCENP-GFP fusion protein, but is caused by Aurora-B activity. We conclude that outer-kinetochore associated Aurora-B activity increases the incidence of lateral kinetochores in monopolar spindles that mimic an early mitotic spindle configuration.

Congression resolves Aurora-B induced lateral attachments. We tested if constitutive Aurora-B activity would increase the incidence of lateral kinetochores in bipolar spindles that allow biorientation and inter-kinetochore tension. To study bipolar spindles, we treated cells with MG132, a proteasome inhibitor. MG132 treated cells expressing Mis12-INCENP-GFP displayed a mixture of both congressed and uncongressed kinetochores (Supplementary Fig. 2A-C), as previously reported ${ }^{26}$. In cells with congressed KTs, we observed KT-MT attachments that were properly bioriented (Supplementary Fig. 2A, insets) and predominantly end-on attached, with a small but statistically significant fraction displaying lateral attachments (Supplementary Fig. 2D). Thus, bipolar spindles partially rescue the end-on conversion defect induced by constitutive Aurora-B activity. We confirmed this finding by quantifying Astrin-SKAP recruitment at kinetochores: although the intensity of KT-associated SKAP was reduced by $\sim 2.5$-fold in Mis12-INCENP-GFP expressing cells compared to non-expressing cells, in agreement with previous report $^{43}$ (Supplementary Fig. 2E and F), SKAP was clearly enriched on congressed and bioriented kinetochore pairs. Thus, bipolar spindles, which allow chromosome congression and biorientation of KT-MT attachments, are able to overcome the end-on conversion defect induced by Aurora-B at the outerkinetochore. 
a mKate2-Tubulin
Mis12-
INCENP(TAA)-GFP


b
Fate of lateral and end-on KTs



C



\section{Lateral}
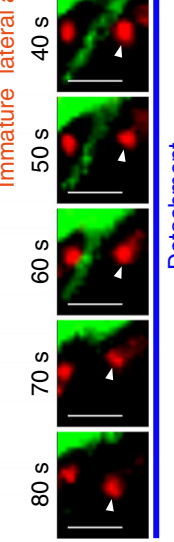

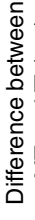

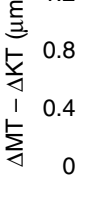

asynch

$-$
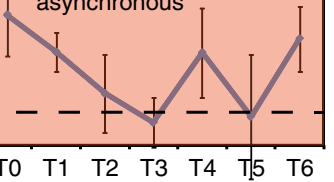

Time (10 $s$ intervals)

Mis12-INCENP(TAA)-GFP $($ Cells $=3 ; \mathrm{KTs}=6$ )

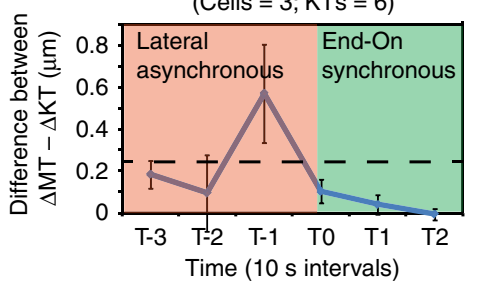

d

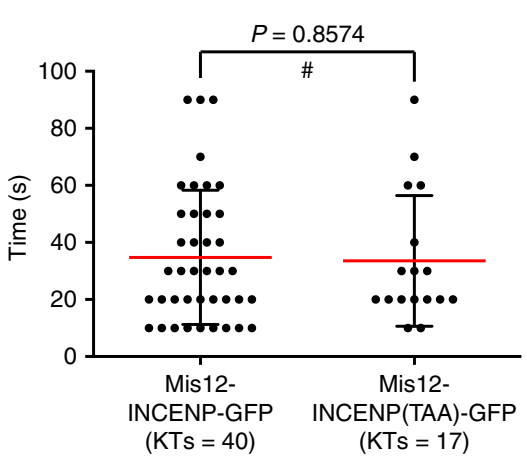

e



Legend:

Low Aurora-B allows end-on attachment

Kinetochore

Microtubule (MT)

AurB activity (high to low)

Fig. 2 Constitutive Aurora-B activity at the outer-kinetochore allows lateral attachments but disrupts end-on conversion. a Single-plane time-lapse images of Z-stacks show the fate of a lateral KT (red) attached to MT (green). Time-lapse images of HeLa cells expressing mKate2-Tubulin and either Mis12INCENP-GFP or Mis12-INCENP (TAA)-GFP mutant were acquired in the presence of Monastrol. Yellow arrows mark shrinking lateral K-fibre and white arrows show the synchronous movement of the KT with MT-end. White arrowheads mark the KT tracked. For three final time-points (left panels) 'intensified' images are included to highlight the K-fibre. Scale bar: $2 \mu \mathrm{m}$. b Graphs show the percentage of lateral (left) or end-on (right) kinetochores that transitioned into other attachment states (D-detached, L-lateral and E-end-on) in time-lapse movies as in a. c Graph shows the difference between $\Delta M T$ (change in MT-end position) and $\Delta K T$ (change in KT position) through time, used to define synchronous or asynchronous movements. Values less than $0.25 \mu m$ (marked by dashed line), sustained for at least $30 \mathrm{~s}$, are indicated as synchronous (green) movement between the KT and MT-end. All other values are indicated as asynchronous movements of the KT and MT-end. d Graph shows the time spent by lateral kinetochores on MT-walls, before changing into another attachment state, in cells expressing either Mis12-INCENP-GFP or Mis12-INCENP (TAA)-GFP. Red and black bars mark mean-time and SD values, respectively, across kinetochores in three experiments. Error bars in $\mathbf{b}$ and $\mathbf{c}$ are SEM values across experiments $\mathbf{b}$ or across kinetochores $\mathbf{c}$. '*' and '\#' indicate statistically significant and insignificant differences, respectively, on the basis of $P$-values obtained across three experimental repeats using a Proportion test $\mathbf{b}$ or unpaired Student's $t$-test $\mathbf{d}$. e Cartoon shows how Aurora-B at the outer-kinetochore disrupts the end-on conversion process by detaching lateral kinetochores prior to end-on conversion. Aurora-B at the outer-kinetochore does not interfere with the capture or maintenance of kinetochores on microtubule walls. Reducing Aurora-B activity allows the formation of mature end-on attachments 
Aurora-B at outer-kinetochore abrogates end-on conversion. To understand the precise reason for the accumulation of lateral attachments in Mis12-INCENP-GFP expressing cells, we investigated three possibilities: (i) whether lateral kinetochores are due to excessive error-correction (i.e., detachment of end-on kinetochores promoting their recapture onto microtubule walls), (ii) abrogation of the process of converting lateral attachments into end-on attachments or (iii) excessive stabilization of lateral KTMT interactions. To distinguish between all three mechanisms, we performed time-lapse microscopy of monopolar spindles of cells co-expressing mKate2-Tubulin and Mis12-INCENP-GFP. We tracked the fate of lateral and end-on kinetochores and
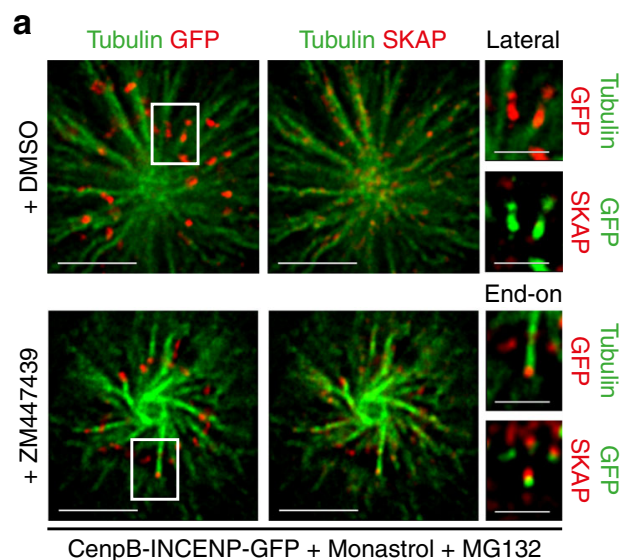

CenpB-INCENP-GFP + Monastrol + MG132

C



b

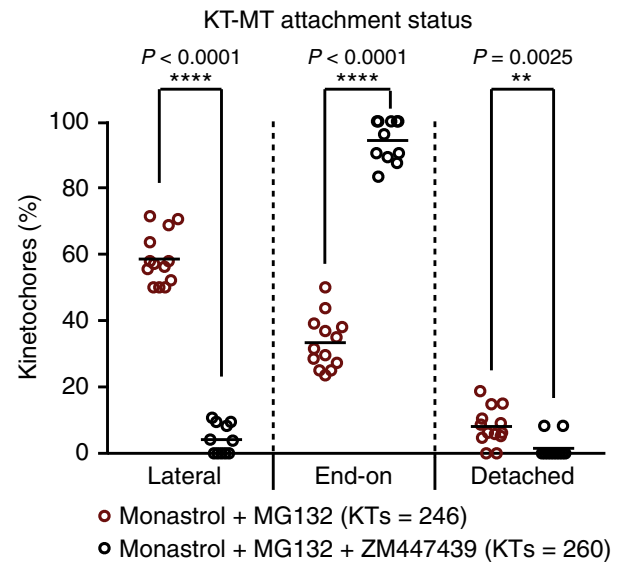

d

Fate of lateral or end-on kinetochores
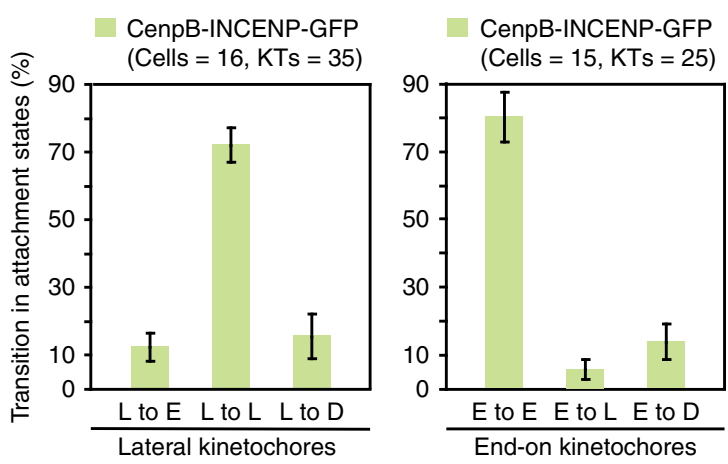

e

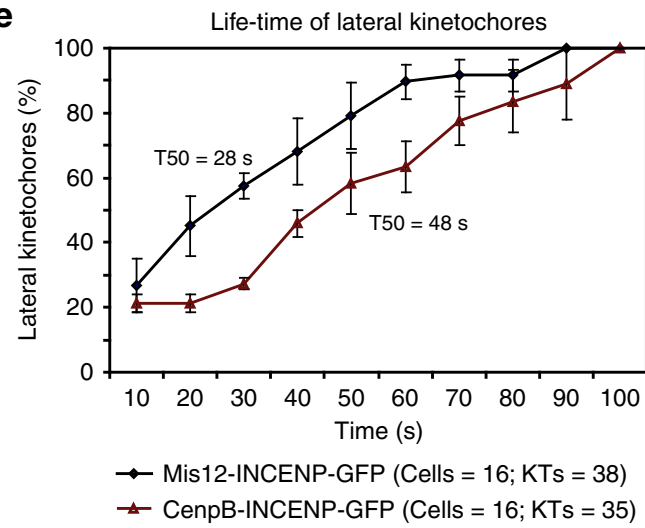

f

\begin{tabular}{|c|c|c|c|c|c|c|}
\hline $\begin{array}{l}\text { Fusion } \\
\text { construct }\end{array}$ & $\begin{array}{l}\text { Constitutive } \\
\text { AurB } \\
\text { localization }\end{array}$ & $\begin{array}{l}\text { Active } \\
\text { AurB }\end{array}$ & $\begin{array}{l}\text { KT-MT } \\
\text { attachment } \\
\text { status }\end{array}$ & $\begin{array}{l}\text { Fate of } \\
\text { lateral KT }\end{array}$ & $\begin{array}{l}\text { Conversion into } \\
\text { non-productive } \\
\text { end-on } \\
\text { attachment }\end{array}$ & $\begin{array}{l}\text { End-on } \\
\text { conversion }\end{array}$ \\
\hline $\begin{array}{l}\text { CenpB- } \\
\text { INCENP } \\
\text {-GFP }\end{array}$ & Cen & $\begin{array}{l}\text { Cen } \\
\text { alone }\end{array}$ & $\begin{array}{l}\text { Lateral } \\
\text { +End-on }\end{array}$ & $\begin{array}{l}\text { Stable } \\
\text { attachment }\end{array}$ & Frequent & $\begin{array}{l}\text { Severely } \\
\text { Reduced }\end{array}$ \\
\hline $\begin{array}{l}\text { Mis12- } \\
\text { INCENP } \\
\text {-GFP }\end{array}$ & $\begin{array}{l}\text { Outer } \\
\text { KT }\end{array}$ & $\begin{array}{l}\text { Outer KT } \\
\text { and Cen }\end{array}$ & $\begin{array}{l}\text { Predominantly } \\
\text { Lateral }\end{array}$ & $\begin{array}{l}\text { Detach } \\
\text { \& recapture } \\
\text { laterally }\end{array}$ & Infrequent & $\begin{array}{l}\text { Severely } \\
\text { Reduced }\end{array}$ \\
\hline
\end{tabular}


analysed changes in the plane of KT-MT interaction and KT movements. A Mis12-INCENP(TAA)-GFP mutant with compromised Aurora-B activity ${ }^{26}, 44-46$ was used as a negative control. In control cells expressing Mis12-INCENP(TAA)-GFP, lateral kinetochores bound to microtubule walls were converted into end-on kinetochores as expected (Fig. 2a). Quantitative analysis of the fate of kinetochores in these control cells showed that the vast majority of lateral kinetochores became end-tethered within $50 \mathrm{~s}$ (Fig. 2b), following which they underwent forward and regressing movements, which were synchronous with changes in K-fibre length (Fig. 2a (left panels) and c (lower panel)). Such synchronous movement between the kinetochore and MT-end demonstrates the establishment of a productive and mature KT-MT attachment that are capable of converting the change in K-fibre length into kinetochore movement. In stark contrast, in cells expressing Mis12-INCENP-GFP, majority of lateral kinetochores failed to convert into end-on kinetochores (Fig. 2b). In these cells, kinetochore movements were not synchronous with changes in K-fibre length (Fig. 2a (right panels) and $c$ (upper panel)), demonstrating an immature KT-MT attachment state. A significant proportion of lateral kinetochores in Mis12-INCENP-GFP expressing cells underwent detachment (Fig. 2a (right panels) and Fig. 2b (left panel)) and reattached onto microtubule walls (Fig. 2a (right panels)). However, the lifetime of lateral kinetochores was not significantly different in Mis12-INCENP-GFP and Mis12-INCENP(TAA)GFP expressing cells (Fig. 2d). This unaltered life-time of lateral kinetochores shows that Aurora-B at the outer-kinetochore does not prohibit the capture or the maintenance of lateral kinetochores. Based on our data on KT-MT attachment status, $\mathrm{KT}$ movements and KT life-time in live-cells, we conclude that Aurora-B activity allows kinetochore capture and maintenance along microtubule walls but it specifically disrupts the end-on conversion process.

Finally, as KT fate analysis showed that end-on (monotelic) kinetochores remained tethered to MT-ends and did not detach in Mis12-INCENP-GFP expressing cells (Fig. 2b, right panel), we are able to rule out excessive error-correction (detachment of end-on kinetochores) as an indirect cause for increase in lateral kinetochores. In summary, we reveal a novel role for Aurora-B in selectively abrogating the end-on conversion process, without disrupting the establishment or maintenance of lateral attachments (Fig. 2e). We conclude that this novel role of Aurora$\mathrm{B}$ in influencing the plane of KT-MT attachment in human cells is independent of the kinase's established role in the errorcorrection process.
Distinct Aurora-B pools disrupt end-on conversion differently. Because active Aurora-B is lost from outer-kinetochore and retained only at the inner centromeric region during metaphase $^{28}$, we tested whether centromere localized Aurora-B influences the end-on conversion process similarly to outerkinetochore localized Aurora-B. For this purpose, we performed fixed and live-cell microscopy studies using CenpB-INCENPGFP, wherein the Aurora-B docking domain is fused to a centromeric protein, CenpB ${ }^{26}$. As expected CenpB-INCENP-GFP signals were predominantly centromeric and did not extent beyond inner kinetochore region (Supplementary Fig. 5B). In monopolar spindles of CenpB-INCENP-GFP expressing cells, nearly $60 \%$ of kinetochores were laterally attached and they lacked SKAP of the Astrin-SKAP complex (Fig. 3a,b), suggesting an end-on conversion defect. This lateral attachment phenotype and lack of Astrin-SKAP complex was rescued under two conditions: in monopolar spindles treated with Aurora-B inhibitor (Fig. 3a,b) and in bipolar spindles with congressed chromosomes (Supplementary Fig. 3A and B). We conclude that a local increase in Aurora-B, restricted to centromere and inner kinetochore region, increases the incidence of lateral kinetochores in monopolar spindles and this altered plane of KT-MT interaction is rescued in congressed kinetochores of bipolar spindles.

To determine the impact of centromere bound Aurora-B on lateral kinetochores, we tracked the fate of kinetochores in cells co-expressing mKate2-Tubulin along with either CenpBINCENP-GFP or CenpB-INCENP(TAA)-GFP (negative control $\left.^{26}\right)$. In control cells expressing CenpB-INCENP(TAA)-GFP, the majority of kinetochores were attached to microtubule-ends and stably tethered without detachment episodes (Supplementary Fig. 4A and B). As expected, lateral kinetochores that matured into end-on kinetochores underwent forward and regressing movements, synchronous with changes in the K-fibre length (Supplementary Fig. 4C). In stark contrast, cells expressing CenpB-INCENP-GFP displayed a low incidence of lateral to endon conversion events (Fig. 3c,d). Detachment episodes of lateral or end-on kinetochores were not high (Fig. 3c,d; while 4/4 syntelic KTs detached, only $3 / 21$ monotelic KTs detached within $10 \mathrm{~s}$ ). Quantitative analysis of kinetochore movements in CenpBINCENP-GFP expressing cells showed that lateral kinetochores remained laterally attached and failed to sustain synchronous movements with the MT-ends of the K-fibre (Fig. 3d and Supplementary Fig. 4D), revealing a role for centromere bound Aurora-B in retarding the end-on conversion event.

While both centromeric and outer-kinetochore pools of Aurora-B disrupt end-on conversion, there were two key

Fig. 3 Spatially distinct pools of Aurora-B impede distinct steps of the end-on conversion process. a Images of cells expressing CenpB-INCENP-GFP exposed to Monastrol and MG132 with either ZM447439 or DMSO (solvent control), as indicated, prior to immunostaining with antibodies against Tubulin, SKAP and GFP. Cropped images show lateral (upper row) and end-on (lower row) kinetochores. Scale: $5 \mu \mathrm{m}$ in main and $2 \mu \mathrm{m}$ in cropped images. Boxed areas correspond to cropped images. b Graph shows percentage of lateral, end-on and detached kinetochores in CenpB-INCENP-GFP expressing cells treated as in a. Each circle represents values from one cell. Black bar marks average values from two independent experimental repeats. ${ }^{*}$ indicates statistically significant difference on the basis of $P$-values obtained using unpaired Student's $t$-test. c Single-plane time-lapse images of Z-stacks show the fate of a lateral or end-on KT (red) bound to MT (green). Time-lapse images of HeLa cells expressing mKate2-Tubulin and CenpB-INCENP-GFP treated with Monastrol. White arrowheads mark KT tracked. Yellow arrows mark the tip of lateral K-fibre. Scale: $2 \mu \mathrm{m}$. d Graph shows percentage of lateral or end-on kinetochores that transitioned into other attachment states (D-detached, L-lateral and E-end-on) in time-lapse movies as in c. Error bars are SEM values across three experimental repeats e Cumulative frequency plots show time spent by lateral kinetochores on MT-walls, before changing into another attachment state, in cells expressing either Mis12-INCENP-GFP or CenpB-INCENP-GFP. Error bars are SEM values across three experimental repeats. Note: Mis12-INCENP-GFP curve values were obtained from data presented in Fig. 3d. T50 values are in seconds derived from the cumulative frequency plot. $\mathbf{f}$ Table contrasts the consequence of localizing Aurora-B at the outer kinetochore (KT) vs. centromere (cen), using Mis12-INCENP-GFP and CenpB-INCENP-GFP fusion proteins, respectively. Both fusion proteins disrupt end-on conversion but they differently control the fate of lateral attachments (within yellow box). Active Aurora-B status (Active AurB) was assessed using immuno-staining with antibodies against AuroraB-pThr232 as in Fig. 1a. KT-MT attachment status was obtained from fixed-cell studies. Fate of lateral kinetochore and instances of non-productive end-on attachments and productive end-on conversion are from live-cell movies 
differences: (i) the lifetime of lateral kinetochores was significantly increased in cells expressing CenpB-INCENP-GFP compared to Mis12-INCENP-GFP (Fig. 3e) and (ii) the number of lateral kinetochores that formed non-productive end-on attachments (end-on attachments lasting less than $30 \mathrm{~s}$ ) was at least twofolds more in cells expressing CenpB-INCENP-GFP $\left(n_{\mathrm{KT}}=13 / 34\right)$ compared to Mis12-INCENP-GFP $\left(n_{\mathrm{KT}}=6 / 37\right)$.

In summary, we conclude that a local increase of Aurora- $B$ at the outer-kinetochore detaches lateral attachments prior to end-on conversion, whereas Aurora-B at the centromere retains

Experimental regime

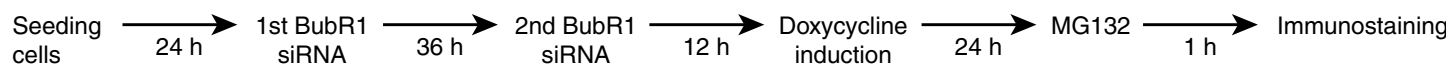

b

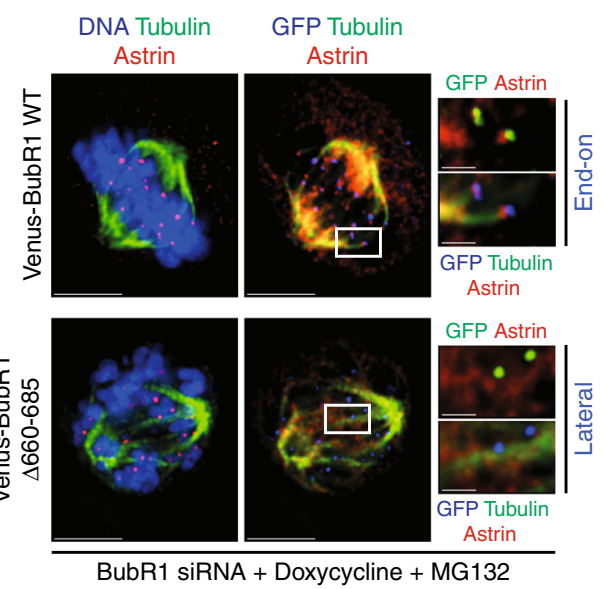

d

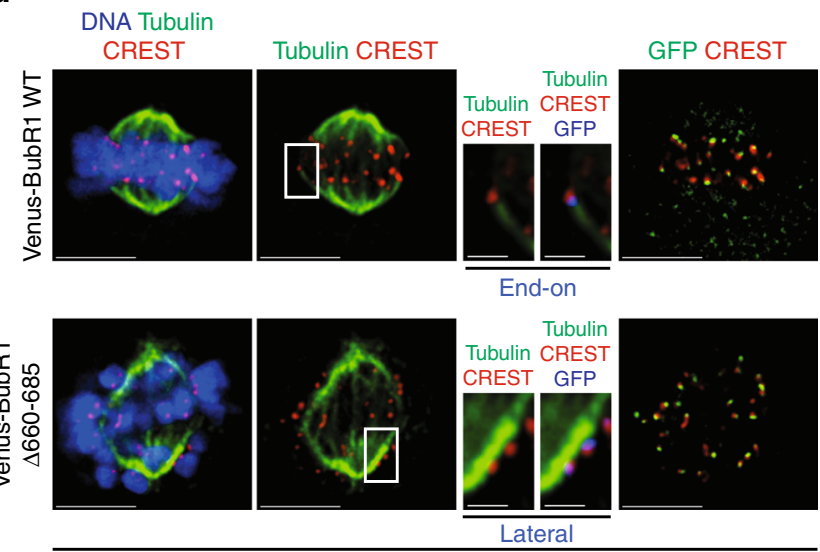

BubR1 siRNA + Doxycycline + MG132

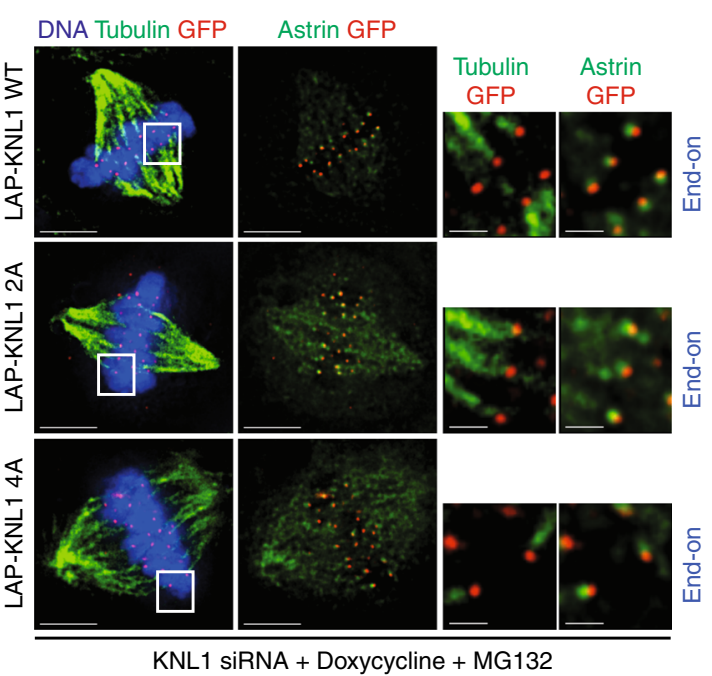

C Astrin intensity at kinetochores

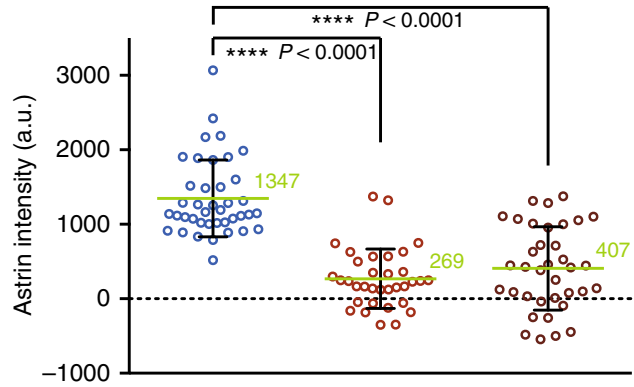

- Venus-BubR1 WT (Cells $=5 ; \mathrm{KTs}=40$ )

- Venus-BubR1 $\triangle 660-685$ congressed (Cells $=5 ; \mathrm{KTs}=34$ )

- Venus-BubR1 $\triangle 660-685$ uncongressed (Cells $=5 ; \mathrm{KTs}=36$ )

e KT-MT attachment status

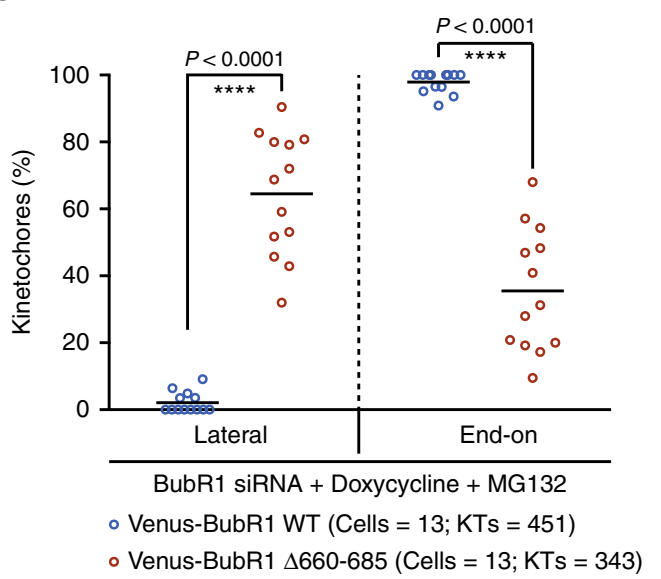

9

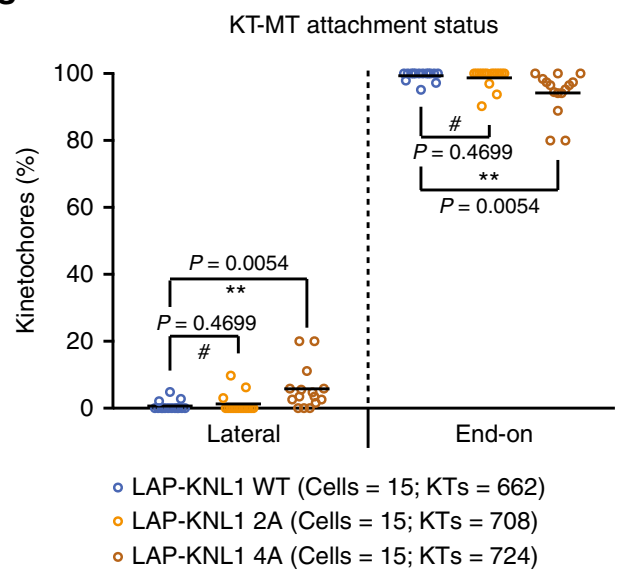


lateral attachments and prevents stable end-on conversion (Fig. 3f). This difference could not be simply explained by gross differences in the extent of fusion protein expression at the kinetochore (Supplementary Fig. 5A) or extent of HEC1 phosphorylation (Supplementary Fig. $5 \mathrm{~B}$ and $\mathrm{C}$ ). We propose that the two different spatial pools of Aurora-B may influence downstream targets differently or to different extent leading to distinct impacts upon the end-on conversion process.

BubR1-PP2A but not KNL1-PP1 controls end-on conversion. We hypothesized that Aurora-B counteracting phosphatases at the outer-kinetochore should be essential for end-on conversion, as Aurora-B activity is high at the outer-kinetochore during early prometaphase ${ }^{47}$. At the outer-kinetochore, two major phosphatases (KNL1 associated PP1 and BubR1 associated PP2A) counteract Aurora-B activity and are needed for the spindle assembly checkpoint or maintaining cold-stable KT-MT attachments ${ }^{30-36}$. The extent to which the two phosphatases control the end-on conversion process has not been studied so far. To address this, we first quantified KT-MT attachment status (end-on vs. lateral) in cells conditionally expressing a Venus (YFP) tagged BubR1 mutant that can not interact with PP2A-B56 (BubR1 $\Delta 660-685)^{31}$ (Fig. 4a). Consistent with previous reports $31,32,48$, our immunostaining studies showed that in cells depleted of endogenous BubR1, the expression of the BubR1 mutant lacking the PP2A docking domain interfered with both PP2A recruitment and chromosome alignment (Supplementary Fig. 6A, C and E and Fig. 4b). We next assessed the enrichment of Astrin-SKAP complex (a marker of mature end-on attachments) at congressed kinetochores in cells treated with MG132. Cells expressing the BubR1 $\Delta 660-685$ mutant failed to recruit Astrin normally in the vast majority of congressed kinetochores, and the kinetochores that displayed Astrin showed a steep fivefold reduction compared to controls (Fig. 4c). Analysis of KT-MT attachment status in MG132-treated BubR1 $\Delta 660-685$ mutant expressing cells demonstrated a highly significant increase in laterally attached kinetochores (Fig. 4d,e) in both congressed and uncongressed kinetochores (Supplementary Fig. 7A). Lateral KT-MT attachment status in BubR1 $\Delta 660-685$ mutant expressing cells was further confirmed using super-resolution microscopy (Supplementary Fig. 7B). These data indicate an impairment of the end-on conversion process in cells lacking BubR1 associated PP2A, despite chromosome congression. Based on the status of KT-MT attachment and Astrin recruitment, we conclude that (i) laterally attached kinetochores predominate in cells lacking BubR1 associated PP2A and (ii) chromosome congression is insufficient to rescue the end-on conversion defect in cells lacking BubR1 associated PP2A-B56.
We next tested if KNL1 associated PP1 phosphatase is required for end-on conversion by comparing KT-MT attachment changes induced by two LAP-tagged KNL1 mutants: (i) KNL1-2A where Aurora-B mediated phosphorylation sites at the PP1 docking motif are mutated to allow constitutive recruitment of PP1 and (ii) KNL1-4A where the PP1 docking RVSF motif is mutated to abrogate PP1 recruitment ${ }^{30,37}$. We first confirmed that PP1 levels are reduced in KNL1 depleted cells expressing LAP-tagged KNL14A compared to LAP-tagged KNL1-WT or LAP-tagged KNL1-2A (Supplementary Fig. 6B and D), as reported ${ }^{37}$. Following MG132 treatment, KNL1 depleted cells expressing either LAP-tagged KNL1-2A or KNL1-WT displayed kinetochores that were congressed and normally end-tethered; whereas, cells expressing LAP-tagged KNL1-4A mutant displayed a mild congression defect (Fig. 4f,g and Supplementary Fig. 6F). A slight increase in lateral kinetochores and reduction in Astrin intensity were observed in cells expressing LAP tagged KNL1-4A mutant, compared to cells expressing either KNL1-2A mutant or KNL1WT fusion proteins (Fig. $4 \mathrm{~g}$ and Supplementary Fig. 6G). However, the percentage of lateral kinetochores and extent of Astrin reduction were much lesser in cells expressing the PP1 docking mutant (KNL1-4A) compared to PP2A docking mutant (BubR1 $\Delta 660-685$ ) (Compare Fig. 4e,g; Fig. 4c and Supplementary Fig. 6G). These studies provide first insight into the extent to which the two phosphatases control the plane of KT-MT interaction and highlight the essential role of BubR1 associated PP2A in controlling the maturation of KT-MT attachments.

Astrin-SKAP is a target of Aurora-B during end-on conversion. Because Aurora-B negatively regulates Astrin-SKAP levels at kinetochores ${ }^{43}$, Astrin-SKAP complex is a potential downstream target of Aurora-B in the end-on conversion process. We quantified KT-MT attachment status in monopolar spindles of Aurora-B inhibited cells depleted of either Astrin or SKAP (Fig. 5a). As expected, in control siRNA treated cells, kinetochores were tethered to MT-ends and were uniformly distant from the spindle poles (Fig. 5b). In contrast, in SKAP siRNA treated cells, kinetochores were dispersed along MT-walls and a reduced proportion of the kinetochores were attached to MT-ends (Fig. 5b and Supplementary Fig. 8A), showing a defect in tethering kinetochores to microtubule-ends despite Aurora-B inhibition. A similar phenotype of increased lateral kinetochores was observed in cells treated with Astrin siRNA despite Aurora-B inhibition (Fig. 5b and Supplementary Fig. 8A). We confirmed that SKAP was absent on the kinetochores of Astrin or SKAP siRNA treated cells (Fig. 5b). We conclude that the Astrin-SKAP complex plays a crucial role in ensuring the correct plane of KT-MT interaction.

\footnotetext{
Fig. 4 BubR1 associated PP2A, but not KNL1 associated PP1, plays a significant role in the end-on conversion process. a Experimental regime: BubR1 siRNA treated HeLa FRT/TO cells conditionally expressing Venus (YFP)-BubR1 (WT or $\triangle 660-685$ mutant) were exposed to Doxycycline for $24 \mathrm{~h}$ and MG132 for $1 \mathrm{~h}$ prior to immunostaining. $\mathbf{b}$ Images of cells treated as in $\mathbf{a}$, immunostained with antibodies against GFP, Tubulin and Astrin and stained with DAPI for DNA. Scale: $5 \mu \mathrm{m}$ in uncropped and $1 \mu \mathrm{m}$ in cropped images. Boxed areas correspond to cropped images. c Graph of Astrin intensities on congressed or uncongressed kinetochores in cells expressing Venus (YFP) tagged -BubR1 WT or $\Delta 660-685$ mutant, as in b. Horizontal lines show average values (in green) across KTs from two independent experiments. Each circle represents values from one kinetochore. $\mathbf{d}$ Images of cells treated as in a, immunostained with antibodies against GFP and Tubulin and CREST anti-sera and stained with DAPI for DNA. Scale: $5 \mu \mathrm{m}$ in uncropped and $1 \mu \mathrm{m}$ in cropped images. Boxed areas correspond to cropped images. e Graph shows percentage of lateral vs. end-on kinetochores in Venus-BubR1 (WT or 4660-685 mutant) expressing cells treated as in d. Each circle represents values from one cell. Black bar marks average values from four independent experiments. $\mathbf{f}$ Images of KNL1 siRNA treated HeLa cells expressing LAP-tagged KNL1 WT or mutants (2A or 4A). Following plasmid transfection, cells were exposed to Doxycycline for $1 \mathrm{~h}$ and then incubated in Doxycycline-free media for $30 \mathrm{~h}$. Prior to fixation cells were exposed to MG132 for $45 \mathrm{~min}$ and immunostained with antibodies against GFP, Tubulin and Astrin. Scale: $5 \mu \mathrm{m}$ in uncropped and $2 \mu \mathrm{m}$ in cropped images. Boxed areas correspond to cropped images. $\mathbf{g}$ Graph shows percentage of lateral vs. end-on kinetochores in LAP tagged KNL1 (WT, 4A or 2A mutant) expressing cells, treated as in $\mathbf{f}$. Each circle represents values from one cell. Black bar marks average values from three independent experimental repeats. In $\mathbf{c}$, e and $\mathbf{g}$, ${ }^{\star \star \prime}$ and \# indicate statistically significant and insignificant differences, respectively (assessed using $P$-values from unpaired Student's $t$-test)
} 
KT attachments to MT-ends but not MT-walls rely on SKAP. To determine why end-tethering of kinetochores is compromised in SKAP depleted cells, we tracked the fate of lateral kinetochores in time-lapse movies of cells co-expressing CenpB-DsRed and YFP-Tubulin (Fig. 5c). Analysing the fate of kinetochores showed that in control cells, lateral kinetochores converted into end-on kinetochores normally (Fig. 5d). However, in SKAP depleted cells, lateral kinetochores either remained attached to microtubule walls or detached from microtubule walls and failed to convert into end-on kinetochores (Fig. 5d), showing a failure in the end- on conversion process. The lifetime of lateral kinetochores was comparable in control and SKAP depleted cells (Fig. 5e,f), indicating a dispensable role for SKAP in capturing and maintaining kinetochores along microtubule walls. Although the conversion of a lateral kinetochore into an end-on kinetochore was relatively infrequent in SKAP depleted cells compared to control depleted cells (Fig. 5d), end-tethered kinetochores could be observed in SKAP depleted cells (Supplementary Fig. 8A). Tracking the fate of these end-tethered kinetochores in SKAP depleted cells showed that they rarely remained end-tethered for longer than a

Experimental regime

$\underset{\text { treatment }}{\operatorname{siRNA}} \underset{60 \mathrm{~h}}{\longrightarrow} \begin{gathered}\text { Monastrol } \\ + \text { MG132 }\end{gathered} \underset{2 \mathrm{~h}}{\longrightarrow}+$ ZM447439 $\underset{45^{\prime}}{\longrightarrow}$ Immunostaining

b

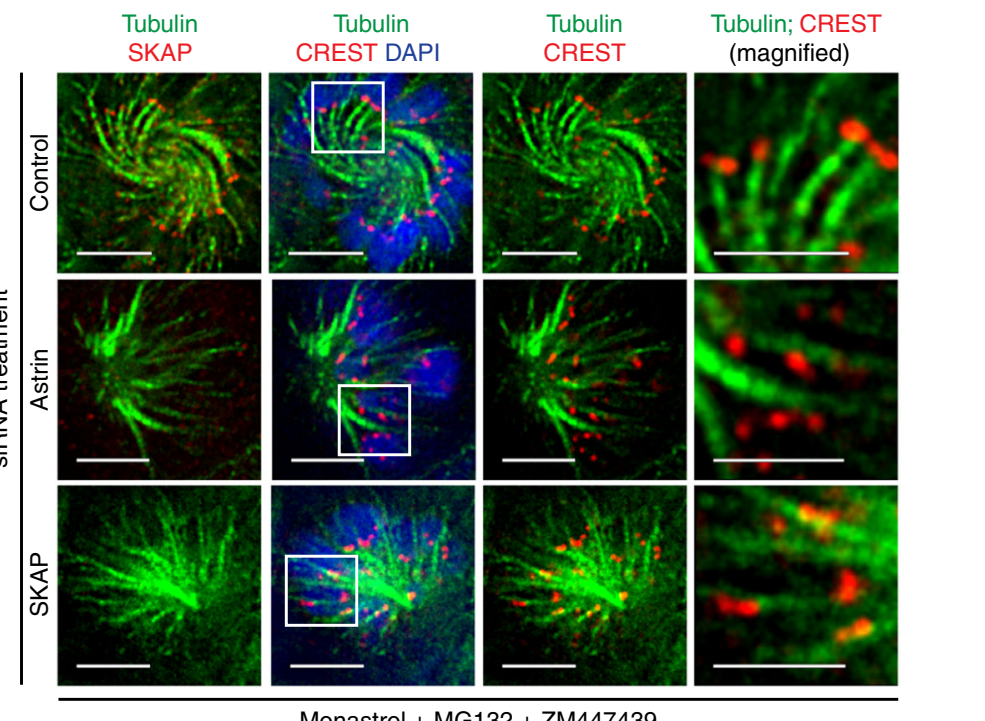

d
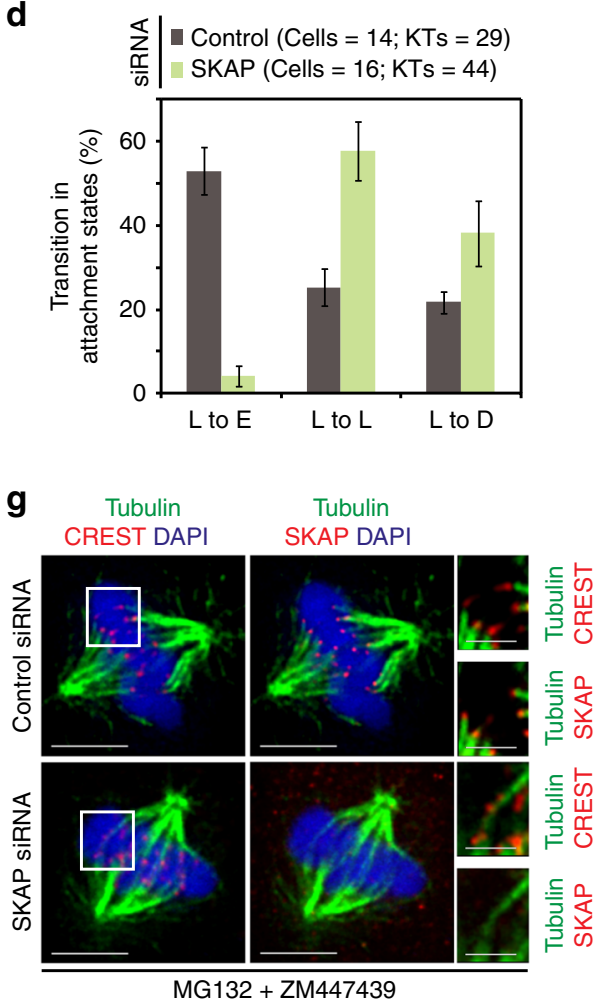

h

Legend: SKAP Kinetochore
C



e



$\mathbf{f}$
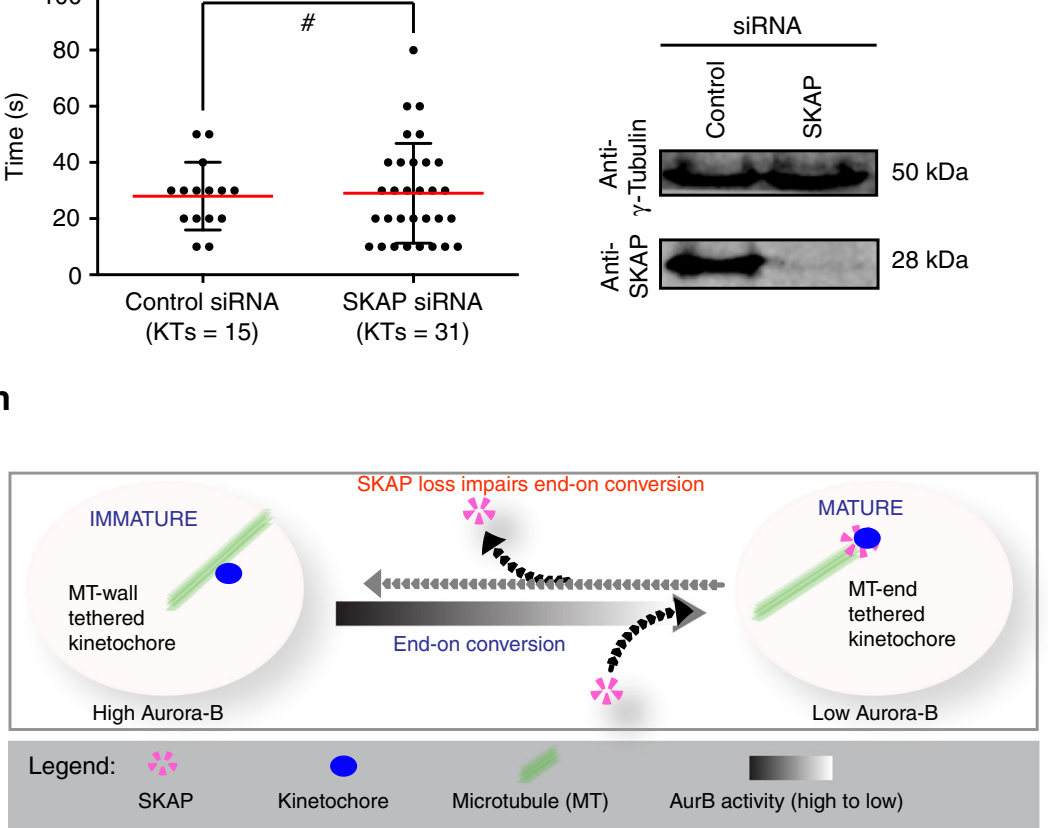

Microtubule (MT)

AurB activity (high to low) 
$30 \mathrm{~s}$, and instead they either became laterally attached or detached $\left(n_{\text {cells }}=4 ; \quad n_{\mathrm{KTs}}=12\right)$. Thus, SKAP facilitates the end-on conversion event and is essential for the stable maintenance of kinetochores at MT-ends but not MT-walls, demonstrating a late role for SKAP in the end-on conversion process.

To confirm that the defect in the plane of KT-MT attachment observed following SKAP depletion is not an siRNA off-target effect, we used a cell line that conditionally expresses an siRNA resistant form of SKAP fused to GFP (GFP-SKAPsiRes $)^{4}, 41$. Following SKAP siRNA treatment, monopolar spindles of GFPSKAP ${ }^{\text {siRes }}$ expressing cells exposed to ZM447439 and MG132 (Supplementary Fig. 8B) displayed end-on attached kinetochores, demonstrating a successful rescue of the SKAP depletion induced lateral attachment phenotype (Supplementary Fig. 8C). Thus, we show the first evidence for SKAP's role in the end-on conversion process, and demonstrate that Aurora-B acts through AstrinSKAP complex to ultimately define the plane of KT-MT attachment (Fig. 5h).

Because chromosome congression partly rescued the endtethering defect caused by constitutive Aurora-B activity, we tested if congression can rescue SKAP depletion induced endtethering defect. Analyzing KT-MT attachments in bipolar spindles following MG132 and ZM447439 treatment showed significantly more lateral kinetochores in SKAP depleted cells, compared to control depleted cells (Fig. $5 \mathrm{~g}$ and Supplementary Fig. 8D). These data show that SKAP is essential for defining the plane of KT-MT interaction in both bipolar and monopolar spindles.

\section{Discussion}

First, we report a novel role for Aurora-B pathway in controlling the plane of KT-MT attachment in human cells. This role for Aurora-B in the maturation of kinetochore-microtubule attachments is distinct from it's well established role in detaching erroneous KT-MT attachments (syntelic or merotelic) $23,25,49$. Second, we show that increasing Aurora-B at the centromere or outer-kinetochore promotes the incidence of lateral attachments by influencing different steps of the end-on conversion process. We find that lateral KT-MT attachments are 'immune' to Aurora$B$ regulation. Third, of the two Aurora- $B$ counteracting phosphatases we tested, BubR1 associated PP2A is more important than KNL1 associated PP1 for defining the plane of KT-MT attachment, revealing functional differences between these two outer-kinetochore associated phosphatases. Finally, we uncover a role for the Astrin-SKAP complex in ensuring the lateral to endon conversion of attachments and maintenance of end-tethered kinetochores. By comparing kinetochores in bipolar and monopolar spindles, we distinguish distinct sequential regulators of the end-on conversion process. Thus, we provide first insight into how the Aurora-B pathway controls the end-on conversion process in human cells.

Our live-cell imaging studies shed light on a physiologically significant puzzle about how human cells protect immature lateral attachments from being destabilized by the errorcorrection enzyme Aurora- $\mathrm{B}^{50}$. We find that increasing centromeric Aurora-B detaches erroneous syntelic (end-on) attachments but spares immature lateral attachments. These data from monopolar spindles show that immature lateral attachments are neither susceptible to Aurora-B's error correction activity nor reliant on biorientation, explaining how early-on attachments could form despite a lack of inter-kinetochore tension. Similar protection of immature lateral attachments against destabilization by Aurora-B/Ipl1 is reported in budding yeast ${ }^{17}$.

In monopolar spindles, which closely mimic early mitotic spindle configuration, we show that Aurora-B activity at the outer-kinetochore allows lateral attachments but blocks end-on conversion. Aurora-B activity at the outer kinetochore is potentially counteracted through BubR1-PP2A that is high on early mitotic kinetochores. Loss of outer-kinetochore associated Aurora-B is likely to tip the balance towards BubR1-associated PP2A phosphatase allowing end-on conversion. Concomitantly, selective enrichment of the Astrin-SKAP complex on MT-end tethered kinetochores ${ }^{4}$, along with the physical separation of centromeric Aurora-B from outer-kinetochore substrates by endon attachment mediated kinetochore pulling, could jointly facilitate the maintenance of a mature end-on attachment (Fig. 6).

Previous studies showed that Astrin is not recruited to uncongressed kinetochores lacking BubR1 associated PP $2 \mathrm{~A}^{48}$. Whether this failure to recruit Astrin is simply due to the lack of congression or directly due to the loss of PP2A was not known. We demonstrate that both Astrin recruitment and end-on attachment status are compromised on congressed kinetochores lacking BubR1 associated PP2A. Thus, BubR1 associated PP2A is required for proper KT-MT attachment even on congressed chromosomes, indicating a new role for BubR1 that is present on metaphase kinetochores ${ }^{51}$. Whether the lack of BubR1-PP2A mediated end-on conversion event(s) is an important reason for higher sensitivity to loss of BubR1 in Brain Tumour Initiating Cells compared to normal cells ${ }^{52}$ would be interesting to explore in the future.

How does the Astrin-SKAP complex mediate end-on conversion? Electron microscopy studies indicate that SKAP ${ }^{159-316}$ : Astrin ${ }^{482-850}$ complex form elongated structures ${ }^{53}$, and Astrin dimers show a 'lollypop' like structure of $80 \mathrm{~nm}$ in length with a flexible hinge ${ }^{54}$. We and others previously showed that a similar hinge region in $\mathrm{Ndc} 80$ (loop domain) is required for tethering kinetochores onto microtubule-ends ${ }^{4,11-13}$. Unlike Ndc80, which

Fig. 5 Astrin-SKAP complex mediates a late step of the end-on conversion process. a Experimental regime: Cells treated with Monastrol and MG132 for $2 \mathrm{~h}$ and ZM447439 for 45 min were immunostained using antibodies against Tubulin and SKAP and CREST antisera. DNA was stained using DAPI.

b Immunofluorescence images of HeLa cells transfected with control, SKAP or Astrin siRNA, as indicated, and treated as in a. Magnified images in the right-most panels correspond to boxed area. Scale bar: $5 \mu \mathrm{m}$. c Single-plane time-lapse images of Z-stacks show the fate of lateral kinetochores (red) attached to MTs (green) in Control or SKAP siRNA treated HeLaYFP-Tub; CenpB-Red cells in Monastrol. White arrowheads mark the KT tracked. Yellow arrows mark shrinking lateral K-fibre and white arrows highlight synchronous movement of the KT and MT-end. Scale bar: $2 \mu$ m. $\mathbf{d}$ Graph shows percentage of lateral kinetochores that remained lateral ( $L$ ) or transitioned into other attachment states (D-detached; E-end-on) in time-lapse movies as in c. Error bars represent SEM from four experiments. e Graph of time spent by lateral kinetochores on MT-walls, before changing into another attachment state, in control or SKAP siRNA treated cells. Red and black bars mark mean-time and SD values, respectively, across kinetochores from four independent experiments. \# indicates statistically insignificant difference (unpaired Student's t-test). f Immunoblots of lysates of cells treated with control or SKAP siRNA harvested after time-lapse microscopy. Antibodies against SKAP and $\gamma$-Tubulin (loading control) were used. $\mathbf{g}$ Images of bipolar spindles with congressed chromosomes in control or SKAP siRNA transfected cells treated with MG132 and ZM447439 and immunostained as in a. Scale bar: $5 \mu$ m in uncropped and $2 \mu \mathrm{m}$ in cropped images. Boxed areas correspond to cropped images. $\mathbf{h}$ Cartoon illustrates SKAP's role in the end-on conversion process: (i) SKAP is selectively recruited to end-on (mature) but not lateral (immature) kinetochores. (ii) SKAP is crucial for the lateral to end-on conversion event but not required for tethering kinetochores onto microtubule walls. Finally, reducing Aurora-B allows end-on attachments in a SKAP dependent manner 


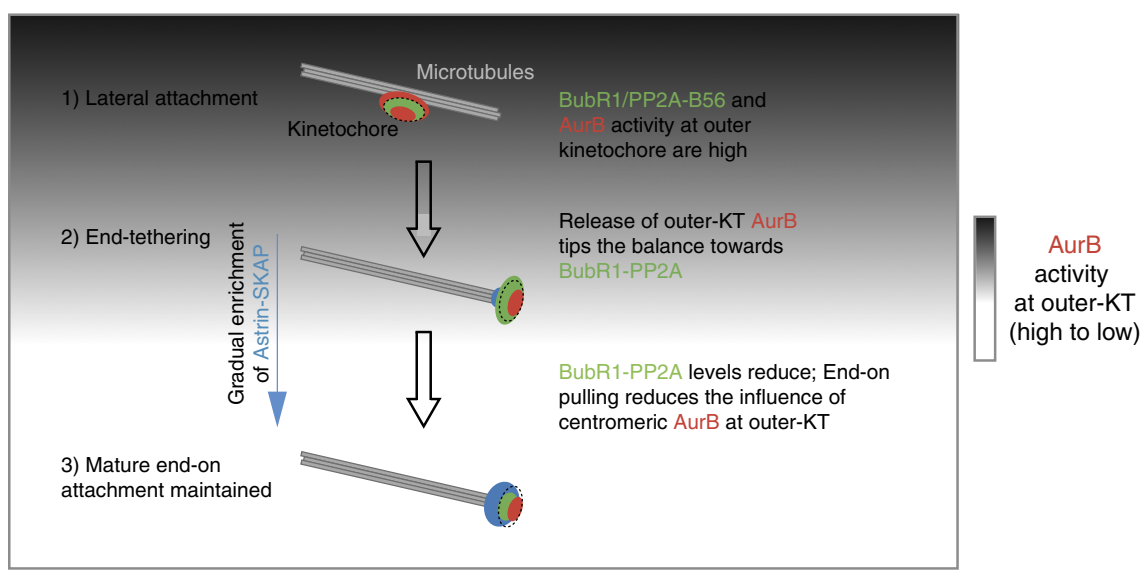

Fig. 6 Schematic description of the end-on conversion process. On laterally attached kinetochores, outer-kinetochore associated Aurora-B kinase and BubR1-PP2A phosphatase levels are high. End-on conversion requires the reduction of outer-kinetochore associated Aurora-B activity, which potentially tips the balance in favour of BubR1-associated PP2A phosphatase allowing the gradual recruitment of Astrin-SKAP complex. Concomitantly, end-tethered kinetochores experience end-on pulling and intra-kinetochore tension, which can spatially separate centromeric Aurora-B from outer-kinetochore substrates. This progressive reduction in Aurora-B activity at the outer-kinetochore will promote further enrichment of Astrin-SKAP complex-a crucial late event in end-on conversion that is essential to maintain mature end-on attachments. Red circles mark outer and inner kinetochore associated Aurora-B, green circles mark BubR1 and blue circles mark Astrin-SKAP

is a core kinetochore protein, the Astrin-SKAP complex is recruited to kinetochores at later stages of mitosis ${ }^{55-57}$ and found predominantly on end-tethered kinetochores in human cells ${ }^{4}$. Future structure studies on Astrin's hinge domain and the kinetochore receptor(s) of Astrin-SKAP complex are likely to reveal crucial insights into how human cells overcome physical barriers in converting the plane of kinetochore-microtubule attachment. Although Astrin-SKAP complex is essential for normal chromosome segregation in human cells ${ }^{41,55-58}$, a truncation mutation in exon-3 of SKAP did not cause somatic defects in mice ${ }^{59}$, suggesting that redundant players may support the loss of SKAP in mice.

In summary, our findings uncover Aurora-B kinase as an upstream regulator of the end-on conversion process. Unlike spindle checkpoint mechanisms that rely on the two phosphatases KNL1-associated PP1 and BubR1-associated PP2A-B56, the end-on conversion process relies significantly on BubR1associated PP2A-B56. Finally, we identify a role for AstrinSKAP in a late event of the end-on conversion process. Thus, we report one of the key molecular pathways that control the plane of kinetochore-microtubule interaction in human cells.

\section{Methods}

Cell culture and synchronization. HeLa cells (ATCC) cultured in Dulbecco's Modified Eagle's Media supplemented with 10\% FCS and antibiotics (Penicillin and Streptomycin) were plated onto glass-bottomed dishes (LabTek) or $13 \mathrm{~mm}$ round coverslips. For inhibition studies, cells were treated with $10 \mu \mathrm{M}$ Monastrol (1305, TOCRIS), $10 \mu \mathrm{M}$ ZM447439 (2458, TOCRIS) or $10 \mu \mathrm{M}$ MG132 (1748, TOCRIS). Inducible expression of GFP-SKAP ${ }^{\text {siRes }}$ was achieved by generating HeLa (GFP-SKAP) as clones obtained from transfecting HeLa FRT/TO cells with pCDNA5-FRT/TO-GFP-SKAPsiRes plasmid vector $^{41}$.

Plasmid and siRNA transfections. siRNA transfection was performed using Oligofectamine according to manufacturers instructions. Briefly, siRNA oligos were incubated in OptiMEM (Invitrogen; 11058-021) and washed 24-36 h prior to analysis. siRNA oligos to deplete Astrin (UCCCGACAACUCACAGAGAAAUU), SKAP (GAAAGAGUCCGAUUCCUAGUU), BubR1 (GAUGGUGAAUUGUGGAAUA) and KNL1 (GCAUGUAUCUCUUAAGGAAUU) were from Dharmacon. Negative control siRNA $(12,935-300)$ was from Invitrogen. HeLa YFPTub; CENPB-Red was generated by transiently transfecting pDsRed-CENPB (Clonetech) into HeLaYFP-Tub used extensively for cell division studies ${ }^{60,61}$. In four well LabTek dishes, $500 \mathrm{ng}$ of plasmid vectors encoding mKate2- $\alpha$ Tubulin (Evrogen) along with plasmid vector encoding Mis12-INCENP-GFP (VSV-Mis12- $\Delta$ Cen INCENP-GFP), Mis12-INCENP(TAA)-GFP (VSV-Mis12- $\Delta$ Cen INCENP(TAA)
GFP), CenpB-INCENP-GFP (VSV-CenpB- $\Delta$ Cen INCENP-GFP) or CenpBINCENP(TAA)-GFP (VSV-CenpB- $\Delta$ Cen INCENP(TAA)-GFP) ${ }^{26}$ were co-transfected into HeLa using Turbofect (R0531, Thermo Scientific) and filmed within $30 \mathrm{~h}$. LAP-tagged KNL1 expression vectors ${ }^{4,37}$ were transiently transfected prior to Doxycycline induction. Following transient transfection of plasmids, cells were assessed after $24 \mathrm{~h}$, except in the case of KNL1 expression plasmids where cells were assessed after $30 \mathrm{~h}$. For SKAP siRNA rescue, Tetracycline (Tet) inducible GFP-SKAP HeLa cells were transfected with SKAP siRNA ${ }^{43}$, $48 \mathrm{~h}$ later treated with tetracycline for 1 and $24 \mathrm{~h}$ later used for studies. siRNA resistant version of GFP-SKAP expression vector (pCDNA5-FRT/TO-GFP-SKAPsiRes) was generated by wobbling nucleotides using point mutagenesis primer GTAAAGCTGGAGATGAAAGAGGAGCGTGTTAGGT TTTTGGAACAGCAAACCTTATG. Plasmid vectors maps are available on request.

Live-cell time-lapse imaging. Cells were transfected with siRNA oligos or plasmid vectors 72 or $24 \mathrm{~h}$, respectively, prior to imaging (unless stated otherwise in experimental regime) and transferred to Leibovitz's L15 medium (Invitrogen; 11415064 ) for imaging at $37^{\circ} \mathrm{C}$. For imaging KT and MT-end dynamics, exposures of $0.04 \mathrm{~s}$ and at least $6 Z$-planes, $0.1 \mu \mathrm{m}$ apart, were acquired every $10 \mathrm{~s}$ for $5 \mathrm{~min}$ using a 100X NA 1.40 oil immersion objective on an Applied Precision DeltaVision Core microscope equipped with a Cascade2 camera under EM mode. Exposure conditions were optimized through long-term high-speed imaging of mKate2-EB3 expressing HeLa cell line ${ }^{62,63}$.

Kinetochore-microtubule attachment status determination. To define KT-MT attachment states, we used the following methodology. First, $360^{\circ}$ rotation of image stacks (3D volume images) generated using Softworx was used to determine the plane of the K-fibre and KT. Second, end-tethered and lateral kinetochores were confirmed on the basis of their synchronous and asynchronous movement, respectively, between the K-fibre tip and the KT. Change in KT and MT-end (MT) positions through time was measured using Softworx distance measurement tool. Values less than $0.25 \mu \mathrm{m}$, sustained for at least $30 \mathrm{~s}$, were marked as synchronous movement of the KT and MT-end. All other values were marked as asynchronous movement of the KT and MT-end. To ensure that the attachment transition measurements are reliable, we only considered end-on kinetochores that remained in end-on state at least for $30 \mathrm{~s}$, lateral kinetochores that remained lateral for greater than $30 \mathrm{~s}$ and detached kinetochores that remained detached for at least $20 \mathrm{~s}$. (In Mis12-INCENP-GFP and mKate2-Tubulin coexpressing cells, we expect a signal overlap since Mis12 resides in the KT outer plate where MT-ends reach ${ }^{64}$ However, in CenpB-DsRed and YFP-Tubulin coexpressing cells, signal overlap is rarely seen). In fixed-cells to set a threshold for high-quality images for quantitative analysis, we excluded cells if greater than $20 \%$ of its kinetochores could not be confidently assigned any KT-MT attachment status. Lateral, end-on or detached kinetochores were scored ${ }^{4}$ and their relative percentages calculated using MS Excel and plotted using Prism Software.

Immunofluorescence, immunoblotting and image analysis. For immunofluorescence, antibodies against $\mathrm{HEC1}^{\mathrm{Ndc} 80} \operatorname{protein}^{65}$ (1:1000), tubulin (Abcam; ab6160; 1:800), pThr232 Aurora-B (Rockland Inc; 600-401-677; 1:500), total 
Aurora-B (Abcam; ab3635; 1:800), GFP (Roche; 1181446001; 1:800), mCherry (Thermo Scientific; M11217; 1:2000), SKAP (Atlas; HPA042027; 1:1000), HEC1pSer44 and HEC1pSer55 ${ }^{28}$ (1:500), Astrin (Novus; NB100-74638; 1:1000), B56 (BD Transduction Laboratories; 610615; 1:500) and CREST antisera (Europa; FZ90C-CS1058; 1:2000) were used. DAPI (Sigma) was used to co-stain DNA. Images of immunostained cells were acquired using 100X NA 1.4 objective on a DeltaVision Core microscope equipped with CoolSnap HQ Camera (Photometrics). Volume rendering (SoftWorx) was performed for 3D analysis of KT-MT attachment status. Deconvolution of live and fixed-cell images and 3D volume rendering were performed using SoftWorx. Quantitative immunoblotting was performed on proteins separated on $12 \%$ SDS-PAGE gels by transferring them overnight onto Nitrocellulose membrane. Membranes were incubated in primary antibodies against Aurora-B pThr232 (Rockland Inc; 600-401-677; 1:1000), SKAP (Atlas; HPA042027; 1:1000) and $\gamma$-Tubulin (Sigma; T6557; 1:800) for an hour and probed using secondary antibodies labeled with infrared fluorescent dyes, which were imaged using an Odyssey imager (Supplementary Fig. 9).

Statistical analysis. To confirm that sampling is sufficient, we used two statistical tests: First, we performed a proportion test or unpaired Student's $t$-test to confirm using $P$-values that we have sampled sufficient number of cells or kinetochores for concluding on differences we report. Second, we measured standard error over mean (SEM) across experimental repeats or across cells and confirmed that the differences we report are not only based on differences in mean values, but also the spread of the mean values between experiments, cells or kinetochores. SD values were obtained across experiments or cells as indicated in the figure legend.

Super-resolution microscopy experiments. For STORM imaging experiments, HeLa cells were transiently transfected with plasmids encoding Venus-BubR1 $\Delta 660-685$ and $24 \mathrm{~h}$ later cells were exposed to MG132 for $1 \mathrm{~h}$. Cells were then prefixed with 4\% PFA in PBS for 20 s, permeabilised with $0.5 \%$ Triton X-100 in PBS for $4 \mathrm{~min}$ and the fixation step was repeated again for $20 \mathrm{~min}$ before quenching with $25 \mathrm{mM}$ glycine for $20 \mathrm{~min}$. One percent BSA in PBS with $0.1 \%$ Tween was used for blocking and all washes were performed using $0.1 \%$ tween in PBS. Cells were immunostained with primary antibodies against $\alpha$-Tubulin (clone B-5-1-2, Sigma-Aldrich; T6074; 1:300) and CREST antisera (Europa; FZ90C-CS1058; 1:2000). For secondary antibody, goat anti-mouse AlexaFluor 647 (Molecular Probes; A21236; 1:800) and donkey anti-human CF680 (Biotium; 20278-1; 1:1000) were used.

Samples were mounted and imaged in a custom-made microscope ${ }^{66}$ and covered with $300 \mu \mathrm{l}$ of imaging buffer $(150 \mathrm{mM}$ Tris- $\mathrm{HCl} \mathrm{pH} 8,10 \%(\mathrm{v} / \mathrm{w})$ glucose, $35 \mathrm{mM}$ cysteamine (MEA), $0.5 \mathrm{mg} / \mathrm{ml}$ glucose oxidase (Sigma; G7141), and $40 \mathrm{mg} / \mathrm{ml}$ catalase (Sigma; C3556)). Typically, 200,000-500,000 frames were recorded. Analysis was performed using custom software written in MATLAB. We acquired two spectral channels simultaneously by splitting the emission with a dichroic (Chroma, T680LPXXR) and assigned the colour based on the relative intensities of the single-molecule localizations in both channels with a cross-talk rate below $0.2 \%{ }^{67}$. Localizations with uncertainties above $25 \mathrm{~nm}$ or a fitted size of the PSF above $170 \mathrm{~nm}$ were discarded. The data were corrected for sample drift using a custom redundant cross-correlation based algorithm. Images were rendered using a Gaussian with a width proportional to the localization precision.

Data availability. The data that support the findings of this study are available from the corresponding author upon reasonable request.

Received: 19 August 2016 Accepted: 12 June 2017

Published online: 28 July 2017

\section{References}

1. Alexander, S. P. \& Rieder, C. L. Chromosome motion during attachment to the vertebrate spindle: initial saltatory-like behavior of chromosomes and quantitative analysis of force production by nascent kinetochore fibers. J. Cell Biol. 113, 805-815 (1991).

2. Tanaka, K. et al. Molecular mechanisms of kinetochore capture by spindle microtubules. Nature 434, 987-994 (2005).

3. Magidson, V. et al. The spatial arrangement of chromosomes during prometaphase facilitates spindle assembly. Cell 146, 555-567 (2011).

4. Shrestha, R. L. \& Draviam, V. M. Lateral to end-on conversion of chromosomemicrotubule attachment requires kinesins CENP-E and MCAK. Curr. Biol. 23, 1514-1526 (2013).

5. Skibbens, R. V., Skeen, V. P. \& Salmon, E. D. Directional instability of kinetochore motility during chromosome congression and segregation in mitotic newt lung cells: a push-pull mechanism. J. Cell. Biol. 122, 859-875 (1993).
6. Cassimeris, L. \& Salmon, E. D. Kinetochore microtubules shorten by loss of subunits at the kinetochores of prometaphase chromosomes. J. Cell Sci 98, 151-158 (1991)

7. Mitchison, T., Evans, L., Schulze, E. \& Kirschner, M. Sites of microtubule assembly and disassembly in the mitotic spindle. Cell 45, 515-527 (1986).

8. Gandhi, S. R. et al. Kinetochore-dependent microtubule rescue ensures their efficient and sustained interactions in early mitosis. Dev. Cell 21, 920-933 (2011).

9. Tanaka, T. U. Kinetochore-microtubule interactions: steps towards biorientation. EMBO J. 29, 4070-4082 (2010).

10. Maure, J.-F. et al. The Ndc80 loop region facilitates formation of kinetochore attachment to the dynamic microtubule plus end. Curr. Biol. 21, 207-213 (2011).

11. Hsu, K.-S. \& Toda, T. Ndc80 internal loop interacts with Dis1/TOG to ensure proper kinetochore-spindle attachment in fission yeast. Curr. Biol. 21, 214-220 (2011).

12. Zhang, G. et al. The Ndc80 internal loop is required for recruitment of the Ska complex to establish end-on microtubule attachment to kinetochores. J. Cell Sci. 125, 3243-3253 (2012).

13. Varma, D. et al. Recruitment of the human Cdt1 replication licensing protein by the loop domain of $\mathrm{Hecl}$ is required for stable kinetochore-microtubule attachment. Nat. Cell Biol. 14, 593-603 (2012).

14. Gachet, Y. et al. Sister kinetochore recapture in fission yeast occurs by two distinct mechanisms, both requiring Dam1 and Klp2. Mol. Biol. Cell 19, 1646-1662 (2008).

15. Gassmann, R. et al. A new mechanism controlling kinetochore-microtubule interactions revealed by comparison of two dynein-targeting components: SPDL-1 and the Rod/Zwilch/Zw10 complex. Genes Dev. 22, 2385-2399 (2008).

16. Cheerambathur, D. K., Gassmann, R., Cook, B., Oegema, K. \& Desai, A. Crosstalk between microtubule attachment complexes ensures accurate chromosome segregation. Science 342, 1239-1242 (2013).

17. Kalantzaki, M. et al. Kinetochore-microtubule error correction is driven by differentially regulated interaction modes. Nat. Cell Biol. 17, 421-433 (2015).

18. Wang, F. et al. Histone H3 Thr-3 phosphorylation by Haspin positions Aurora B at centromeres in mitosis. Science 330, 231-235 (2010).

19. Wang, F. et al. A positive feedback loop involving Haspin and Aurora B promotes CPC accumulation at centromeres in mitosis. Curr. Biol. 21, 1061-1069 (2011).

20. Yamagishi, Y., Honda, T., Tanno, Y. \& Watanabe, Y. Two histone marks establish the inner centromere and chromosome bi-orientation. Science 330, 239-243 (2010).

21. Hauf, S. et al. The small molecule Hesperadin reveals a role for Aurora B in correcting kinetochore-microtubule attachment and in maintaining the spindle assembly checkpoint. J. Cell Biol. 161, 281-294 (2003).

22. Tanaka, T. U. et al. Evidence that the Ipl1-Sli15 (Aurora kinase-INCENP) complex promotes chromosome bi-orientation by altering kinetochore-spindle pole connections. Cell 108, 317-329 (2002).

23. Knowlton, A. L., Lan, W. \& Stukenberg, P. T. Aurora B is enriched at merotelic attachment sites, where it regulates MCAK. Curr. Biol. 16, 1705-1710 (2006).

24. Kallio, M. J., McCleland, M. L., Stukenberg, P. T. \& Gorbsky, G. J. Inhibition of aurora B kinase blocks chromosome segregation, overrides the spindle checkpoint, and perturbs microtubule dynamics in mitosis. Curr. Biol. 12, 900-905 (2002).

25. Cimini, D., Wan, X., Hirel, C. B. \& Salmon, E. D. Aurora kinase promotes turnover of kinetochore microtubules to reduce chromosome segregation errors. Curr. Biol. 16, 1711-1718 (2006).

26. Liu, D., Vader, G., Vromans, M. J. M., Lampson, M. A. \& Lens, S. M. A. Sensing chromosome bi-orientation by spatial separation of aurora B kinase from kinetochore substrates. Science 323, 1350-1353 (2009).

27. Welburn, J. P. I. et al. Aurora B phosphorylates spatially distinct targets to differentially regulate the kinetochore-microtubule interface. Mol. Cell 38, 383-392 (2010).

28. DeLuca, K. F., Lens, S. M. A. \& DeLuca, J. G. Temporal changes in Hec1 phosphorylation control kinetochore-microtubule attachment stability during mitosis. J. Cell Sci. 124, 622-634 (2011).

29. Posch, M. et al. Sds22 regulates aurora B activity and microtubule-kinetochore interactions at mitosis. J. Cell Biol. 191, 61-74 (2010).

30. Liu, D. et al. Regulated targeting of protein phosphatase 1 to the outer kinetochore by KNL1 opposes Aurora B kinase. J. Cell Biol. 188, 809-820 (2010).

31. Kruse, T. et al. Direct binding between BubR1 and B56-PP2A phosphatase complexes regulate mitotic progression. J. Cell Sci. 126, 1086-1092 (2013).

32. Suijkerbuijk, S. J. E., Vleugel, M., Teixeira, A. \& Kops, G. J. P. L. Integration of kinase and phosphatase activities by BUBR1 ensures formation of stable kinetochore-microtubule attachments. Dev. Cell 23, 745-755 (2012).

33. Xu, P., Raetz, E. A., Kitagawa, M., Virshup, D. M. \& Lee, S. H. BUBR1 recruits PP2A via the B56 family of targeting subunits to promote chromosome congression. Biol. Open 2, 479-486 (2013). 
34. Meadows, J. C. et al. Spindle checkpoint silencing requires association of PP1 to both Spc7 and kinesin-8 motors. Dev. Cell 20, 739-750 (2011).

35. Rosenberg, J. S., Cross, F. R. \& Funabiki, H. KNL1/Spc105 recruits PP1 to silence the spindle assembly checkpoint. Curr. Biol. 21, 942-947 (2011).

36. Foley, E. A., Maldonado, M. \& Kapoor, T. M. Formation of stable attachments between kinetochores and microtubules depends on the B56-PP2A phosphatase. Nat. Cell Biol. 13, 1265-1271 (2011).

37. Nijenhuis, W., Vallardi, G., Teixeira, A., Kops, G. J. P. L. \& Saurin, A. T. Negative feedback at kinetochores underlies a responsive spindle checkpoint signal. Nat. Cell Biol. 16, 1257-1264 (2014).

38. Emanuele, M. J. et al. Aurora B kinase and protein phosphatase 1 have opposing roles in modulating kinetochore assembly. J. Cell Biol. 181, 241-254 (2008).

39. Carmena, M., Wheelock, M., Funabiki, H. \& Earnshaw, W. C. The chromosomal passenger complex $(\mathrm{CPC})$ : from easy rider to the godfather of mitosis. Nat. Rev. Mol. Cell Biol. 13, 789-803 (2012).

40. Krenn, V. \& Musacchio, A. The Aurora B kinase in chromosome bi-orientation and spindle checkpoint signaling. Front. Oncol. 5, 225 (2015).

41. Tamura, N. et al. A proteomic study of mitotic phase-specific interactors of EB1 reveals a role for SXIP-mediated protein interactions in anaphase onset. Biol. Open 4, 155-169 (2015).

42. Dunsch, A. K., Linnane, E., Barr, F. A. \& Gruneberg, U. The astrin-kinastrin/ SKAP complex localizes to microtubule plus ends and facilitates chromosome alignment. J. Cell Biol. 192, 959-968 (2011).

43. Schmidt, J. C. et al. Aurora B kinase controls the targeting of the Astrin-SKAP complex to bioriented kinetochores. J. Cell Biol. 191, 269-280 (2010).

44. Honda, R., Körner, R. \& Nigg, E. A. Exploring the functional interactions between Aurora B, INCENP, and survivin in mitosis. Mol. Biol. Cell 14, 3325-3341 (2003).

45. Bishop, J. D. \& Schumacher, J. M. Phosphorylation of the carboxyl terminus of inner centromere protein (INCENP) by the Aurora B Kinase stimulates Aurora B kinase activity. J. Biol. Chem. 277, 27577-27580 (2002).

46. Sessa, F. et al. Mechanism of Aurora B activation by INCENP and inhibition by hesperadin. Mol. Cell 18, 379-391 (2005).

47. Caldas, G. V., DeLuca, K. F. \& DeLuca, J. G. KNL1 facilitates phosphorylation of outer kinetochore proteins by promoting Aurora B kinase activity. J. Cell Biol. 203, 957-969 (2013).

48. Xu, P., Virshup, D. M. \& Lee, S. H. B56-PP2A regulates motor dynamics for mitotic chromosome alignment. J. Cell Sci. 127, 4567-4573 (2014).

49. Lampson, M. A., Renduchitala, K., Khodjakov, A. \& Kapoor, T. M. Correcting improper chromosome-spindle attachments during cell division. Nat. Cell Biol. 6, 232-237 (2004)

50. Khodjakov, A. \& Pines, J. Centromere tension: a divisive issue. Nat. Cell Biol. 12, 919-923 (2010).

51. Taylor, S. S., Hussein, D., Wang, Y., Elderkin, S. \& Morrow, C. J. Kinetochore localisation and phosphorylation of the mitotic checkpoint components Bub1 and BubR1 are differentially regulated by spindle events in human cells. J. Cell Sci. 114, 4385-4395 (2001).

52. Ding, Y. et al. Cancer-Specific requirement for BUB1B/BUBR1 in human brain tumor isolates and genetically transformed cells. Cancer Discov. 3, 198-211 (2013).

53. Friese, A. et al. Molecular requirements for the inter-subunit interaction and kinetochore recruitment of SKAP and Astrin. Nat. Commun. 7, 11407 (2016).

54. Gruber, J., Harborth, J., Schnabel, J., Weber, K. \& Hatzfeld, M. The mitoticspindle-associated protein astrin is essential for progression through mitosis. J. Cell Sci. 115, 4053-4059 (2002)

55. Thein, K. H., Kleylein-Sohn, J., Nigg, E. A. \& Gruneberg, U. Astrin is required for the maintenance of sister chromatid cohesion and centrosome integrity. J. Cell Biol. 178, 345-354 (2007).

56. Mack, G. J. \& Compton, D. A. Analysis of mitotic microtubule-associated proteins using mass spectrometry identifies astrin, a spindle-associated protein. Proc. Natl Acad. Sci. USA 98, 14434-14439 (2001).

57. Fang, L., Seki, A. \& Fang, G. SKAP associates with kinetochores and promotes the metaphase-to-anaphase transition. Cell Cycle 8, 2819-2827 (2009).

58. Dunsch, A. K. et al. Dynein light chain 1 and a spindle-associated adaptor promote dynein asymmetry and spindle orientation. J. Cell Biol. 198, 1039-1054 (2012).

59. Grey, C. et al. SKAP, an outer kinetochore protein, is required for mouse germ cell development. Reproduction 151, 239-251 (2016).
60. Shrestha, R. L. et al. TAO1 kinase maintains chromosomal stability by facilitating proper congression of chromosomes. Open Biol. 4, 130108 (2014).

61. Corrigan, A. M. et al. Automated tracking of mitotic spindle pole positions shows that LGN is required for spindle rotation but not orientation maintenance. Cell Cycle 12, 2643-2655 (2013).

62. Nakai, Y. et al. High-speed microscopy with an electrically tunable lens to image the dynamics of in vivo molecular complexes. Rev. Sci. Instrum. 86, 013707 (2015).

63. Iorio, F. et al. A semi-supervised approach for refining transcriptional signatures of drug response and repositioning predictions. PLOS ONE 10, e0139446 (2015).

64. Ciferri, C. et al. Implications for kinetochore-microtubule attachment from the structure of an engineered Ndc80 complex. Cell 133, 427-439 (2008).

65. Draviam, V. M., Shapiro, I., Aldridge, B. \& Sorger, P. K. Misorientation and reduced stretching of aligned sister kinetochores promote chromosome missegregation in EB1- or APC-depleted cells. EMBO J. 25, 2814-2827 (2006).

66. Schoen, I., Ries, J., Klotzsch, E., Ewers, H. \& Vogel, V. Binding-activated localization microscopy of DNA structures. Nano Lett. 11, 4008-4011 (2011).

67. Früh, S. M., Schoen, I., Ries, J. \& Vogel, V. Molecular architecture of native fibronectin fibrils. Nat. Commun. 6, 7275 (2015).

\section{Acknowledgements}

We thank J. DeLuca for phospho-specific HEC1 antibodies, S. Lens for Mis12-INCENPGFP and CenpB-INCENP-GFP expression vectors, A. Saurin for KNL1 expression vectors and J. Nilsson for BubR1-expressing cell lines. We thank the Draviam group members and in particular Madeleine Hart for comments on the manuscript. We also thank S. Court, P. Ungerer, U. Matti and A. Sossick for technical and infrastructural support. D.C. is supported through an MRC studentship award (MR/K50127X/1; RG70550) and Cambridge Commonwealth, European and International Trust. This work was supported by research funding from Cancer Research UK Career (C28598/A9787), MRC (MR/K50127X/1; RG70550), Royal Society (JP100104) and QMUL laboratory startup grant.

\section{Author contributions}

D.C. contributed to data in Fig. 4, Supplementary Figs. 5, 6 and 7, and the illustration of model in Fig. 6. R.A.R. and N.T. contributed to Supplementary Fig. 1A and reagents in Supplementary Fig. 8B, respectively. J.R., D.C. and K.C. contributed to super-resolution images in Supplementary Fig. 7B. R.L.S. contributed to data in all other figures. Manuscript text was written by V.M.D. and revised together with R.L.S., D.C. and N.T.

\section{Additional information}

Supplementary Information accompanies this paper at doi:10.1038/s41467-017-00209-Z.

Competing interests: The authors declare no competing financial interests.

Reprints and permission information is available online at http://npg.nature.com/ reprintsandpermissions/

Publisher's note: Springer Nature remains neutral with regard to jurisdictional claims in published maps and institutional affiliations.



Open Access This article is licensed under a Creative Commons Attribution 4.0 International License, which permits use, sharing, adaptation, distribution and reproduction in any medium or format, as long as you give appropriate credit to the original author(s) and the source, provide a link to the Creative Commons license, and indicate if changes were made. The images or other third party material in this article are included in the article's Creative Commons license, unless indicated otherwise in a credit line to the material. If material is not included in the article's Creative Commons license and your intended use is not permitted by statutory regulation or exceeds the permitted use, you will need to obtain permission directly from the copyright holder. To view a copy of this license, visit http://creativecommons.org/ licenses/by/4.0/.

(c) The Author(s) 2017 